\title{
Essential Metabolic Routes as a Way to ESKAPE from Antibiotic Resistance
}

\author{
1 Angélica Luana C. Barra ${ }^{1}$, Lívia de Oliveira C. Dantas ${ }^{1}$, Luana Galvão Morão ${ }^{1}$, Raíssa F. \\ 2 Gutierrez ${ }^{1}$, Igor Polikarpov ${ }^{1}$, Carsten Wrenger ${ }^{2 *}$ and Alessandro S. Nascimento ${ }^{1 *}$ \\ $3{ }^{1}$ São Carlos Institute of Physics, University of Sao Paulo. Av. Trabalhador São-carlense, n 400, São \\ 4 Carlos, SP. Brazil. \\ $5 \quad 2$ Department of Parasitology, Institute of Biomedical Sciences, University of Sao Paulo. Av. Prof. \\ 6 Lineu Prestes, 1374 - Cidade Universitária, São Paulo, SP, Brazil. \\ 7 * Correspondence: \\ 8 Corresponding Authors \\ 9 cwrenger@icb.usp.br (CW) or asnascimento@ifsc.usp.br (ASN)
}

10 Keywords: ESKAPE pathogens, thiamine, pyridoxal 5'-phosphate, antibiotic resistance.

12 Abstract

13 The antibiotic resistance is a worldwide concern that requires a concerted action from physicians, 14 patients, governmental agencies and academia to prevent infections and the spread of resistance, to track resistant bacteria, to improve the use of current antibiotics and to develop new antibiotics. Despite the efforts spent so far, the current antibiotics in the market are restricted to only five general targets/pathways highlighting the need for basic research focusing on the discovery and evaluation of new potential targets. Here we interrogate two biosynthetic pathways as potentially druggable pathways in bacteria. The biosynthesis pathway for thiamine (vitamin B1), absent in humans, but found in many bacteria, including organisms in the group of the ESKAPE pathogens (Enterococcus faecium, Staphylococcus aureus, Klebsiella pneumoniae, Acinetobacter baumanii, Pseudomonas aeruginosa and Enterobacter species) and the biosynthesis pathway for pyridoxal 5'-phosphate and its vitamers (vitamin B6), found in S. aureus. Using current genomic data, we discuss the possibilities of inhibition of enzymes in the pathway and review the current state of the art in the scientific literature. 
Antibiotic resistance is an urgent threat to human health and requires urgent actions from physicians, patients, industries, governmental agencies and the academic community worldwide. According to the last document from the Centers for Disease Control and Prevention (CDC) regarding antibiotic resistance in the United States, from 2013, the number of people with serious infections caused by resistant bacteria reaches two million every year, with at least 23,000 deaths per year directly caused by these infections (1). The situation is similarly warning in Europe, where 25,100 deaths were reported from the European Centre for Disease Prevention and Control in 2007 (2). Globally, 700,000 deaths are estimated every year as a consequence of antibiotic resistance (3). The same CDC document lists four general action lines to address antibiotic resistance: $(i)$ preventing infections and the spread of resistance; (ii) tracking resistant bacteria; (iii) improving the use of current antibiotics; (iv) developing of new antibiotics (1).

Since bacteria have a short doubling time and efficient mechanisms for plasmid sharing, the development of antibiotic resistance is a very efficient defense mechanism. So, as previously said by Walsh and Wencewicz, the development of resistance is not a matter of if, but rather a matter of when (4) and, despite the title of this paper (which is rather provocative), there is no way we can escape from it (5). On the contrary, the development/discovery of new antibiotics will tend to be a continuous goal to be achieved in drug discovery pipelines.

Interestingly, even after what has been named as the 'golden age' of antibiotics development, the drugs currently in the market are restricted to only five molecular targets and/or pathways (4): $(i)$ the peptidoglycan/cell wall biosynthesis, site of action of beta-lactam antibiotics, for example; ( $i i)$ the protein biosynthesis, where the ribosome is an important target; (iii) DNA replication and RNA transcription; (iv) the folate biosynthesis pathway, and $(v)$ the disruption of the bacterial membrane. Given the relevance of the antibiotic therapy and the emergence of antibiotic resistance, the available choices of current drugs are very narrow in the context of the mechanism of action restricted to only five molecular/pathway targets.

Antibiotic resistance, as a threat to human health, should be addressed in multiple, and simultaneous ways. For academia, an interesting way to address antibiotic resistance is the discovery and validation of new targets/pathways that could be specifically targeted by new antibiotic candidates. It is worthy of note that, according to Kelly and Davies (3), no new class of antibiotics was discovered and released for routine treatment since the 1980s, highlighting the outstanding role that academia can have in the preliminary research for discovery and validation of druggable targets/pathways.

About $7 \%$ of the E. coli genome, 303 genes, were shown to be composed of essential genes $(5,6)$. Under stress conditions caused by a limited medium, additional 119 genes show some condition-dependent essentiality, including several genes related to the metabolism and synthesis of essential molecules such as amino acids, and nucleotides (5) and, obviously, some of these targets/pathways can be an interesting choices for the development of new antibiotic candidates. A promising approach to screen compounds for their activity in metabolic pathways was shown by Zlitni and coworkers (7). The authors searched for antibacterial compounds under poor nutrient media and found three potential compounds in this screening strategy (7). Worth of note, the metabolic profile of existing antibiotics showed that the supplementation of vitamins B5, B6, B1, and B2 did not significantly reverse the antimicrobial effect of any of the 24 inhibitors assayed (7), suggesting that the current antibiotics do not explore these essential pathways. 
In this context, the enzymatic routes for the biosynthesis of vitamins are very interesting pathways to be explored as potential targets for the discovery of new antibiotic candidates. Vitamin B1, for example, cannot be synthesized by humans, although several microorganisms can synthesize this vitamin, including pathogens. In the absence of thiamin (vitamin B1), the activity of several carbohydrate metabolism enzymes is impaired, including pyruvate dehydrogenase, which connects glycolysis and the citric acid cycle (8). Other thiamin dependent enzymes are transketolase, $\alpha$ ketoacid decarboxylase, $\alpha$-ketoacid dehydrogenase and acetolactate synthase (9). A very similar scenario was observed for pyridoxal 5'-phosphate (vitamin B6) in the model Gram-positive organism Bacillus subtilis (10).

The pathways involved in the synthesis of vitamins, in particular, vitamin B1 (thiamin) and B6 (pyridoxal), seem to be of great relevance, since they are involved in central processes in the metabolism of carbohydrates and amino acids and the corresponding enzymes are found in most bacteria, fungi and plants but not in humans (8), favoring the development of specific drugs with minimal side effects due to the interaction with the host. However, a few questions still stand: (i) how feasible are the targets involved in the pathways for the biosynthesis of thiamin and pyridoxal for the microorganisms with a higher emergency in terms of resistance, or ESKAPE: Enterococcus faecium, Staphylococcus aureus, Klebsiella pneumoniae, Acinetobacter baumanii, Pseudomonas aeruginosa and Enterobacter species? (ii) What enzymes are present and what do we know about them?

Here we used the available genomic and proteomic data to address these questions focusing on the ESKAPE pathogens. Vitamins B1 and B6 were chosen for this analysis, since the biosynthetic pathways for these enzymes have already been validated as molecular targets for some human pathogens, such as thiamin for Plasmodium falciparum (11), or pyridoxal for P. falciparum (12) or Trypanosoma brucei (13), for example.

\section{Methods}

The analyses provided here are the result of the interrogation of the enzymes in the biosynthesis pathways for thiamin and pyridoxine phosphate or pyridoxal phosphate using the KEGG database $(14,15)$. For this purpose, the KEGG pathway for thiamin metabolism (map 00730) was listed for the ESKAPE pathogens using, whenever possible, commercial strains rather than specific or antibiotic-resistant strains. Briefly, for each pathogen (Enterococcus faecium, Staphylococcus aureus, Klebsiella pneumoniae, Acinetobacter baumanii, Pseudomonas aeruginosa and Enterobacter) a KEGG search for the maps of thiamin and pyridoxal metabolism was done, looking for the existing genes in commercial strains for each of the ESKAPE organisms. For the sake of comparison, some additional resistant strains were listed, with no differences in the observed genes for the pathways under study. A similar search was carried out for the vitamin B6 metabolism (map 00750) in KEGG, interrogating the existing enzymes for the ESKAPE pathogens in comparison with humans. The analysis was focused in the enzymes in the biosynthesis of B1 and B6 vitamin in an attempt to identify the most promising targets for future medicinal chemistry studies. Finally, a table listing the existing genes for each pathogen was compiled and is presented in the following sections.

Using the existing literature, we sought to identify whenever possible the cases of enzymes with functional redundancy with another enzyme for the same organism. The identified cases were discussed as less promising targets for medicinal chemistry campaigns. Additionally, the existing 
113 data for enzyme inhibition using natural or synthetic compounds was compiled to provide initial

114 proof-of-concept clues about the druggability of the identified promising targets.

115 When necessary, the sequence of an enzyme of the pathway was used to search for homologues using 116 BLAST (16) searches against the PDB (17) or the non-redundant database of proteins using BLAST 117 default parameters, i.e., minimum expected threshold of 10, a word size of 6 and the BLOSUM62 118 substitution matrix.

\section{Results and Discussion}

\section{$121 \quad 3.1 \quad$ Thiamin Biosynthesis}

The biosynthesis pathway for thiamin involves two branches, as shown in Figure 1. In summary, thiamin is synthesized from 4-amino-5-hydroxymethyl-2- methylpyrimidine (HMP, superior branch in Figure 1) and 5-(2-hydroxyethyl)-4-methylthiazole (THZ, inferior branch). Both compounds are phosphorylated by ThiD and ThiM, respectively. Finally, the enzyme ThiE, central to the pathway, is responsible to synthesize thiamine phosphate (TMP) by merging the two branches (18). Thiamin phosphate can be dephosphorylated to thiamine and then pyrophosphorylated to thiamin diphosphate (TPP) by TPK. Alternatively, thiamin can be degraded to HMP and THZ by TenA, a thiaminase II enzyme.

According to the KEGG pathway database, humans lack many of the enzymes of this pathway, but not all of them. In humans, a TPK (TPK1) enzyme is found, with UNIPROT ID Q9H3S4. Other than that, the remaining enzymes in the biosynthetic pathway for thiamin are missing in humans, making them very attractive for the design of chemical probes that could be used as proof-of-concept compounds.

The analysis of the thiamin pathway for Enterococcus faecium (ATCC 8459) in the KEGG database did not identify genes for ThiE, ThiM and TenA. For this species, only ThiD and TPK were positively identified. However, a BLAST search for TenA homologous within the non-redundant database restricted to Enterococcus faecium (TAXID 1352) identified a single result for thiaminase II (GenBank: SAM74984.1), four results for ThiE (SAZ10134.1, WP_086323306.1, WP_010732763.1 and WP_072538983.1) and two results for ThiM (SAZ10238.1, EJY48288.1). The small number of hits in the BLAST search may suggest some issues in the annotation.

Interestingly, when the Enterococcus faecalis thiamin pathway is compared with the pathway observed for E. faecium, many differences are found. The KEGG pathway for E. faecalis (ATCC 29212) shows that the entire pathway, as described in Figure 1 is found: ThiD, ThiM, ThiE, TPK and TenA, in contrast with E. faecium. Additionally, there is no change in the repertoire of enzymes in the pathway for the Vancomycin-resistant strain V583 as compared to the ATCC 29121 strain.

For Staphylococcus aureus (NCTC 8325) and Klebsiella pneumoniae (subsp. pneumoniae ATCC 43816 KPPR1), the KEGG pathway indicates that all the enzymes in the pathway are observed, with no changes to an $S$. aureus resistant strain such as COL (MRSA) as compared to the NCTC 8325. For Acinetobacter baumannii (ATCC 14978), all enzymes are observed but TPK. Instead, thiamin phosphate may be converted directly to thiamin diphosphate by a thiaminmonophosphate kinase, and then converted to thiamin triphosphate by an adenylate kinase. In the 
153 case of Pseudomonas aeruginosa (NCGM 1900), some enzymes are missing: ThiD, TenA, TPK. 154 Finally, Enterobacter sp. (638) has ThiD, ThiE, ThiM and misses TenA and TPK.

The overall panel of enzymes for the thiamin pathway for the ESKAPE pathogens is summarized in Table 1.

TenA was shown to play a dual role in thiamine synthesis and salvage (19): beyond its

In the other branch of the thiamin biosynthesis pathway, thiazole phosphate can be generated from thiazole alcohol incorporated from the medium and phosphorylated by ThiM or it can be synthesized in a series of enzymatically catalyzed reactions using amino acids such as glycine or tyrosine as substrates (9). In this context, according to Begley and coworkers (9), lesions in the ThiM gene were observed to be prototrophic. In contrast, selective mutations of ThiD and ThiE led to the requirement of externally provided thiamin (9).

ThiE is central to the pathway since the enzyme merges the two branches to finally synthesize thiamin phosphate (Figure 1). It is important to highlight that many of the mutational analysis was carried out in E. coli and, as Table 1 shows, there is significant variance among bacterial species.

Taking together, the analysis of the literature suggests that ThiD and ThiE may represent interesting targets for the development of chemical probes to further evaluate their function. TPK is expressed in humans and the inhibition of this enzyme could lead to harmful effects on the human host. TenA inhibition could be overcome by the somewhat functional redundancy with thiC (thiA) and ThiM function can be dispensable since thiazole phosphate can be synthesized de novo.

ThiD and ThiE are found in most ESKAPE organisms. Exceptions are P. aeruginosa, which lacks ThiD and E. faecium that lacks ThiE, according to KEGG data. For ThiE, in particular, it was reported that 4-amino-2-trifluoromethyl-5-hydromethylpyrimidine $\left(\mathrm{CF}_{3}-\mathrm{HMP}\right)$, an $\mathrm{HMP}$ analogue, can be converted by ThiD to $\mathrm{CF}_{3}-\mathrm{HMP}$ pyrophosphate, which in turn inhibits ThiE $(8,21)$. This enzymatic inhibition culminated with the inhibition of E. coli growth (8), suggesting that this strategy might be promising for the development of new inhibitors.

In terms of the structural biology, some crystal structures of ThiD are available by the time of the writing of this paper, including the enzymes from Salmonella enterica (PDB ID 1JXH (22)), Clostridioides difficile (PDB ID 4JJP), B. subtilis (PDB ID 2I5B (23)), Thermus thermophilus (PDB ID 1UB0), A. baumannii (PDB ID 4YL5), Bacteroides thetaiotaomicron (PDB ID 3MBH) and the bifunctional enzyme from Saccharomyces cerevisiae (PDB ID 3RM5). Typically, ThiDs are folded as typical ribokinases, with a central 8 -stranded $\beta$-sheet surrounded by $8 \alpha$-helices and structural studies suggest that some surface loops may have a structural change based on the presence of the nucleotide (23).

Interestingly, the $S$. aureus pyridoxal kinase enzyme ( $S a \mathrm{PdxK})$ has a dual role, phosphorylating pyridoxal and pyridoxine in the pyridoxal de novo biosynthesis pathway as well as HMP in the thiamin biosynthesis pathway, with a $\mathrm{K}_{M}$ almost 20 times greater for HMP than for 
pyridoxal and $\mathrm{k}_{\text {cat }}$ values 3 times faster for pyridoxal (24). So, SaPdxK has some redundancy with $S$. aureus ThiD (SaThiD), with less efficiency, though.

SaThiD was shown to be potentially inhibited by Rugulactone ( $\mathrm{Ru} 0)$, a natural product as well as by its derivatives $\mathrm{Ru} 1$ and $\mathrm{Ru} 2$, with $\mathrm{IC}_{50}$ values ranging from 14 to $32 \mu \mathrm{M}(25)$. In the absence of thiamine in the medium, the MIC observed for Ru1 was four times lower than in the presence of thiamine for L. monocytogenes (25), suggesting that the inhibitory effect of Rugulactone is due to ThiD inhibition, although Rugulactone also inhibits other kinases.

For ThiE, the crystal structures of a few enzymes are available, including the enzyme from Pyrococcus furiosus (PDB ID 1XI3), B. subtilis (PDB IDs 1G4T, 3O15, 3016, 1G69, 1G4E, 1G67, 1G4P, 1YAD (26)), Mycobacterium tuberculosis (3O63) and for the bifunctional enzyme from Candida glabrata (PDB IDs 3NL2 and 3NM1 (27)). No crystal structure of an ESKAPE pathogen ThiE is available to date. From the structural point of view, ThiE is typically folded as an $\alpha / \beta$ TIM barrel, with thiamine binding at the top of the barrel, as elegantly shown in the set of crystal structures determined for the B. subtilis enzyme (28).

At this point, an important question has to be addressed: can the blockade of thiamine de novo biosynthesis pathway be overcome by internalization of the vitamin? In principle, several microorganisms can import thiamine from the environment (29). So, how effective can be the inhibition of ThiD and ThiE particularly for pathogenic bacteria? This question can be addressed in several levels. On the first level, the essentiality of ThiD, for example, has been shown some pathogenic microorganisms (23), such as Streptococcus pneumoniae (30), Haemophilus influenzae (31) and Mycobacterium tuberculosis (32). This is not the case for other bacteria. For B. subtilis, for example, ThiD gene was shown to be dispensable (23). Whether ThiD and ThiE are essential for the ESKAPE pathogens is still an open question to be addressed by further investigation. On a second level, Nodwell and coworkers showed that Listeria monocytogenes, which is known to uptake thiamine from the environment, is still sensible to Rugulactone and its derivatives Ru1 and Ru2 (25). When grown in a chemical defined media without thiamine, the inhibitory effects of Rugulactone are potentiated with a 4-fold reduction in the MIC (25). However, the inhibitory effect observed even in the presence of thiamine highlight that the blockade of the de novo biosynthesis pathway is still a promising strategy. As a third level, the natural compound bacimethrin, isolated from Bacillus megaterium and from Streptomyces albus is known to be toxic for bacteria with MIC at low micromolar range $(33,34)$. The mechanism of action of bacimethrin is based on the formation of 2'methoxy-thiamine where bacimethrin is used by ThiD instead of HMP. 2'-methoxy-thiamine pyrophosphate inhibits $E$. coli growth at concentrations 15 times lower than bacimethrin (35). Interestingly, some Salmonella enterica thiD mutants were shown to be bacimethrin resistant (36). Together, these data suggest that a very promising approach can be devised by the design of suicide drugs, i.e., compounds that are recognized by the enzymes in the pathway but lead to final compounds that can't be used as thiamine substituents. Bacimethrin is a good example of a suicide inhibitor and, although its effects can be reverted by increased thiamine concentrations, the vitamin concentration that pathogens are exposed are usually defined within a narrow range. 
Pyridoxal, pyridoxine and pyridoxamine, together with their respective phosphate esters compose the six vitamers for vitamin B6 and can be interconverted (37). There are two biosynthetic routes for pyridoxal 5'-phosphate: a deoxyxylulose 5-phosphate-dependent pathway found in some bacteria and a deoxyxylulose 5-phosphate-independent pathway found in all kingdoms $(37,38)$. This second and widespread pathway, curiously, depends on only two enzymes, as shown in Figure 2.

In this pathway, the enzyme Pdx2 converts L-glutamine into L-glutamate and an ammonium

Similar to what is observed to the thiamine biosynthesis pathway, humans lack the genes for Pdx1 and Pdx2, making the development of specific antibiotic candidates an attracting strategy. In the same direction, for some pathogenic organisms such as Helicobacter pylori (44), Mycobacterium tuberculosis (45), and Streptococcus pneumoniae (46), the depletion in vitamin B6 resulted in reduced virulence, indicating that the biosynthesis of this vitamin may be a good strategy for the design of new antibiotic candidates.

Very interestingly and in contrast to what was observed for the thiamine pathways, from the ESKAPE pathogens, $S$. aureus is the only microorganism that has the ribose 5-phosphate dependent pathway for the biosynthesis of pyridoxal 5'-phosphate, as shown in Table 2. A single enzyme (Pdx1 or Pdx2 alone) is never observed for these organisms (Table 2).

A comparison between the pyridoxal/pyridoxine phosphate pathways for E. faecium and $E$. faecalis shows that both pathogens miss the Pdx enzymes. On the other hand, some other pathogenic organisms, including organisms with high antibiotic resistance rates, have already been identified as susceptible to modulations of the pyridoxal phosphate biosynthesis pathway. Some organisms which have been the focus of basic research include Plasmodium falciparum and Plasmodium vivax, the malaria pathogens (47-50).

In terms of the structural biology of the enzymes in the vitamin B6 biosynthesis pathway, the S. aureus Pdx1 enzyme (UNIPROT ID Q2G0Q1) is a close homologue of Bacillus subtilis Pdx1 (81\% identity, PDB ID 2NV1 (42)), Geobacillus stearothermophilus Pdx1 (78\% identity, PDB ID 1ZNN (51)) and Thermus thermophilus enzyme (67\% identity, PDB ID 2ZBT). S. aureus Pdx2, has Geobacillus kaustophilus (PDB ID 4WXY (40)), Geobacillus stearothermophilus (PDB ID 1Q7R) and B. subtillis (PDB IDs 2NV0 and 2NV2 (42)) enzymes as its close homologues, with 60\%, 60\% and $58 \%$ identity in sequence similarity respectively.

In terms of its structure, the Pdx1 enzyme has a typical $(\beta / \alpha)_{8}$ barrel fold with a central 8 stranded $\beta$-barrel surrounded by $8 \alpha$-helices (51). The most impressing feature in the Pdx1 enzyme structure is its quaternary arrangement, where six Pdx1 enzymes interact with each other to form a 'donut-like' arrangement with about $100 \AA$ in diameter. For Pdx2, a Rossman fold is observed with an $\alpha / \beta / \alpha$ sandwich topology (42). Curiously, in the active PLP synthase complex, 12 Pdx1 molecules (a two-layer donut) interact with $12 \mathrm{Pdx} 2$ enzymes. However, Pdx2 molecules do not interact with each other in this complex (42). 
Using a structural homology model, Reeksting and coworkers identified some ribose 5'phosphate analogues with interesting in vitro inhibitory effects on the enzyme Pdx1 from $P$. falciparum (12). The compounds were identified in a structure-based computational screening campaign and were shown to have inhibitory effects in vivo, as well as the in vitro effect. The authors also showed that the in vivo effects could be at least partially suppressed in a mutant strain overexpressing $\mathrm{Pdx} 1$ and $\mathrm{Pdx} 2$, in a clear indication that the effect of the analogues was due their inhibition of the pyridoxal 5'-phosphate biosynthesis pathway (12).

The inhibition of the Pdx2 activity by the glutamine analogue acivicin was also demonstrated to be a feasible strategy for the inhibition of the vitamin B6 biosynthesis pathway (52). Raschle and coworkers showed that when inhibited by acivicin, Pdx2 is incapable of interacting with Pdx1, thus disrupting the pyridoxal 5'-phosphate synthase activity. Interestingly, acivicin is a covalent inhibitor, that binds to a cysteine residue. So, it seems that multiple strategies may be available for the design of new binders, including the inhibition of Pdx2 (covalent and non-covalent), the inhibition of Pdx1 and possibly the inhibition of the assembly of the 24-mer complex with $\mathrm{Pdx} 1 / \mathrm{Pdx} 2$. Another possibility to address the inhibition of PLP biosynthesis might be at the level of the Pdx1 transcription, which was shown to be regulated by PdxR (46). In principle, if PdxR action could be regulated by an exogenous chemical probe, the PLP synthase activity would be regulated as well. However, a proof-of-concept experimental validation is still necessary.

Finally, a salvage pathway for vitamin B6 is found in many bacteria, as well as in humans. In E. coli, it involves two enzymes: PdxK and PdxY. These kinases act phosphorylating the vitamers pyridoxal, pyridoxine and pyridoxamine into their phosphorylated forms $(23,37,53)$. Interestingly, in some organisms, including pathogenic microorganisms such as $S$. aureus, the PdxK activity is carried out by the HMP kinase ThiD, showing some functional convergence between the vitamin B6 and B1 pathways (24). Not surprisingly, this enzyme has also been shown to be validated druggable target for some pathogens, such as Trypanosoma brucei for example (13), although specificity may be an important issue, since humans also have genes for PdxK known to be inhibited by drugs like theophylline, for example, with known neurotoxic effects (54).

Again, the same question asked for the thiamine biosynthesis pathway can be asked here: how can the microbial uptake of vitamin B6 overcome the blockade of the biosynthetic pathway? Some evidences suggest that the blockade might be effective. For example, in $S$. pneumoniae, the deletion of Pdx1 resulted in defective growth of the bacteria. The growth was shown to be restored under increased concentrations of vitamin B6 (46). A similar scenario was also observed for $M$. tuberculosis (45), suggesting that the uptake might not be enough to meet the pathogen's requirement of the vitamin.

In conclusion, the biosynthesis pathways for vitamin B1 (thiamine) and B6 (pyridoxal 5'phosphate) may be interesting molecular targets for the development of new chemical probes aiming to inhibit the synthesis of these essential cofactors in pathogenic organisms. Model compounds, mainly based on substrate analogues showed that inhibition of the vitamin B1 biosynthetic enzymes, ThiD and ThiE, as well as $\mathrm{Pdx} 1, \operatorname{Pdx} 2$ and $\mathrm{PdxK}$ in pyridoxal 5'-phosphate biosynthesis pathway have shown an initial proof of concept for the model and it is up the scientific community and/or researcher in industry to explore these target further.

\section{Conflict of Interest}


319 The authors declare that the research was conducted in the absence of any commercial or financial

320 relationships that could be construed as a potential conflict of interest.

\section{Author Contributions}

$322 \mathrm{CW}$, IP and ASN conceived the project. LGM and RFG analyzed the thiamine pathway, while ALCB 323 and LOCD analyzed the pyridoxal pathway. All authors wrote and approved the manuscript.

\section{Funding}

325

Financial support was provided by Fundação de Amparo à Pesquisa do Estado de São Paulo 326 (FAPESP) through grants 2017/18173-0, 2015/26722-8 and 2015/13684-0 and by Conselho Nacional 327 de Desenvolvimento Científico e Tecnológico (CNPq), through grants 303165/2018-9 and 328 406936/2017-0.

\section{$3297 \quad$ Acknowledgments}

330 The authors thank the funding agencies FAPESP and CNPq. We are also indebted to Maria 331 Auxiliadora M. Santos, Lívia Regina M. Margarido, Josimar Sartori and João Fernando Possatto for 332 their technical support. This manuscript has been released as a Pre-Print at BioRxiv (55). 


\section{References}

336 1. CDC. Antibiotic resistance threats in the USA. (2013)

337 2. WHO. Prioritization of Pathogens to Guide Discovery, Research and Development of New

$338 \quad$ Antibiotics for Drug-Resistant Bacterial Infections, Including Tuberculosis. (2017)

339 doi:10.1192/bjp.111.479.1009-a

340 3. Kelly R, Davies SC. Tackling antimicrobial resistance globally. Med J Aust (2017) 207:371-

341 373.e1. doi:10.5694/mja17.00865

342 4. Walsh CT, Wencewicz T a. Prospects for new antibiotics: a molecule-centered perspective. $J$

$343 \quad$ Antibiot (Tokyo) (2013) 67:7-22. doi:10.1038/ja.2013.49

344 5. Brown ED, Wright GD. Antibacterial drug discovery in the resistance era. Nature (2016)

$345 \quad$ 529:336-343. doi:10.1038/nature17042

6. Baba T, Ara T, Hasegawa M, Takai Y, Okumura Y, Baba M, Datsenko KA, Tomita M, Wanner BL, Mori H. Construction of Escherichia coli K-12 in-frame, single-gene knockout mutants: The Keio collection. Mol Syst Biol (2006) doi:10.1038/msb4100050

7. Zlitni S, Ferruccio LF, Brown ED. Metabolic suppression identifies new antibacterial inhibitors under nutrient limitation. Nat Chem Biol (2013) 9:796-804.

351

352 doi:10.1038/nchembio.1361

8. Du Q, Wang H, Xie J. Thiamin (vitamin B1) biosynthesis and regulation: A rich source of antimicrobial drug targets? Int J Biol Sci (2011) 7:41-52. doi:10.7150/ijbs.7.41

9. Begley TP, Downs DM, Ealick SE, McLafferty FW, Van Loon APGM, Taylor S, Campobasso N, Chiu HJ, Kinsland C, Reddick JJ, et al. Thiamin biosynthesis in prokaryotes. Arch Microbiol (1999) 171:293-300. doi:10.1007/s002030050713

12. Reeksting SB, Müller IB, Burger PB, Burgos ES, Salmon L, Louw AI, Birkholtz LM, Wrenger C. Exploring inhibition of Pdx1, a component of the PLP synthase complex of the human malaria parasite Plasmodium falciparum. Biochem J (2013) 449:175-187. doi:10.1042/BJ20120925

13. Jones DC, Alphey MS, Wyllie S, Fairlamb AH. Chemical, genetic and structural assessment of pyridoxal kinase as a drug target in the African trypanosome. Mol Microbiol (2012) 86:51-64. doi:10.1111/j.1365-2958.2012.08189.x 
370

372

373

374

375

376

377

378

379

380

381

382

383

384

385

386

387

388

389

390

391

392

393

394

395

396

397

398

399

400

401

402

403

404

405

406

407

14. Kanehisa M. KEGG: Kyoto Encyclopedia of Genes and Genomes. Nucleic Acids Res (2000) 28:27-30. doi:10.1093/nar/28.1.27

15. Kanehisa M, Furumichi M, Tanabe M, Sato Y, Morishima K. KEGG: New perspectives on genomes, pathways, diseases and drugs. Nucleic Acids Res (2017) 45:D353-D361. doi:10.1093/nar/gkw1092

16. Camacho C, Coulouris G, Avagyan V, Ma N, Papadopoulos J, Bealer K, Madden TL. BLAST+: Architecture and applications. BMC Bioinformatics (2009) 10:1-9. doi:10.1186/1471-2105-10-421

17. Berman HM, Battistuz T, Bhat TN, Bluhm WF, Bourne PE, Burkhardt K, Iype L, Jain S, Fagan P, Marvin J, et al. The Protein Data Bank. Acta Crystallogr Sect D-Biological Crystallogr (2002) 58:899-907. doi:10.1107/S0907444902003451

18. Backstrom AD, McMordie RAS, Begley TP. Biosynthesis of Thiamin I: The Function of the thiE Gene Product. J Am Chem Soc (1995) 117:2351-2352. doi:10.1021/ja00113a025

19. Begum A, Drebes J, Kikhney A, Müller IB, Perbandt M, Svergun D, Wrenger C, Betzel C. Staphylococcus aureus thiaminase II: Oligomerization warrants proteolytic protection against serine proteases. Acta Crystallogr Sect D Biol Crystallogr (2013) 69:2320-2329. doi:10.1107/S0907444913021550

20. Jenkins AH, Schyns G, Potot S, Sun G, Begley TP. A new thiamin salvage pathway. Nat Chem Biol (2007) 3:492-497. doi:10.1038/nchembio.2007.13

21. Reddick JJ, Nicewonger R, Begley TP. Mechanistic Studies on Thiamin Phosphate Synthase: Evidence for a Dissociative Mechanism $\dagger$. Biochemistry (2001) 40:10095-10102. doi:10.1021/bi010267q

22. Cheng G, Bennett EM, Begley TP, Ealick SE. Crystal structure of 4-amino-5-hydroxymethyl2-methylpyrimidine phosphate kinase from Salmonella typhimurium at $2.3 \AA$ resolution. Structure (2002) 10:225-235. doi:10.1016/S0969-2126(02)00708-6

23. Newman JA, Das SK, Sedelnikova SE, Rice DW. The Crystal Structure of an ADP Complex of Bacillus subtilis Pyridoxal Kinase Provides Evidence for the Parallel Emergence of Enzyme Activity During Evolution. J Mol Biol (2006) 363:520-530. doi:10.1016/j.jmb.2006.08.013

24. Nodwell MB, Koch MF, Alte F, Schneider S, Sieber SA. A subfamily of bacterial ribokinases utilizes a hemithioacetal for pyridoxal phosphate salvage. J Am Chem Soc (2014) 136:4992_ 4999. doi:10.1021/ja411785r

25. Nodwell MB, Menz H, Kirsch SF, Sieber SA. Rugulactone and its Analogues Exert Antibacterial Effects through Multiple Mechanisms Including Inhibition of Thiamine Biosynthesis. ChemBioChem (2012) 13:1439-1446. doi:10.1002/cbic.201200265

26. Peapus DH, Chiu HJ, Campobasso N, Reddick JJ, Begley TP, Ealick SE. Structural characterization of the enzyme-substrate, enzyme-intermediate, and enzyme-product complexes of thiamin phosphate synthase. Biochemistry (2001) 40:10103-10114. doi:10.1021/bi0104726 
408

409

410

411

412

413

414

415

416

417

418

419

420

421

422

423

424

425

426

427

428

429

430

431

432

433

434

435

436

437

438

439

440

441

442

443

444

445

27. Paul D, Chatterjee A, Begley TP, Ealick SE. Domain organization in Candida glabrata THI6, a bifunctional enzyme required for thiamin biosynthesis in Eukaryotes. Biochemistry (2010) 49:9922-9934. doi:10.1021/bi101008u

28. Peapus DH, Chiu H-J, Campobasso N, Reddick JJ, Begley TP, Ealick SE. Structural Characterization of the Enzyme-Substrate, Enzyme-Intermediate, and Enzyme-Product Complexes of Thiamin Phosphate Synthase $\dagger, \ddagger$ Biochemistry (2001) 40:10103-10114. doi:10.1021/bi0104726

29. Manzetti S, Zhang J, van der Spoel D. Thiamin Function, Metabolism, Uptake, and Transport. Biochemistry (2014) 53:821-835. doi:10.1021/bi401618y

30. Hava DL, Camilli A. Large-scale identification of serotype 4 Streptococcus pneumoniae virulence factors. Mol Microbiol (2002) 45:1389-1406. doi:10.1046/j.1365-2958.2002.t01-103106.x

31. Akerley BJ, Rubin EJ, Novick VL, Amaya K, Judson N, Mekalanos JJ. A genome-scale analysis for identification of genes required for growth or survival of Haemophilus influenzae. Proc Natl Acad Sci U S A (2002) 99:966-971. doi:10.1073/pnas.012602299

32. Sassetti CM, Boyd DH, Rubin EJ. Comprehensive identification of conditionally essential genes in mycobacteria. Proc Natl Acad Sci U S A (2001) 98:12712-12717. doi:10.1073/pnas.231275498

33. Reddick JJ, Saha S, Lee J, Melnick JS, Perkins J, Begley TP. The mechanism of action of bacimethrin, a naturally occurring thiamin antimetabolite. Bioorg Med Chem Lett (2001) 11:2245-2248. doi:10.1016/S0960-894X(01)00373-0

34. Breiding-Mack S, Zeeck A, Zahner H. Metabolic Products Of Microorganisms. 239 Bacimethrin Isolated From Streptomyces Albus Identification, Derivatives, Synthesis And Biological Properties. J Antibiot (Tokyo) (1987) 40:1431-1439. doi:10.7164/antibiotics.40.1431

35. Reddick JJ, Saha S, Lee J, Melnick JS, Perkins J, Begley TP. The mechanism of action of bacimethrin, a naturally occurring thiamin antimetabolite. Bioorg Med Chem Lett (2001) 11:2245-2248. doi:10.1016/S0960-894X(01)00373-0

36. Zilles JL, Croal LR, Downs DM. Action of the Thiamine Antagonist Bacimethrin on Thiamine Biosynthesis. J Bacteriol (2000) 182:5606-5610. doi:10.1128/JB.182.19.5606-5610.2000

37. Mukherjee T, Hanes J, Tews I, Ealick SE, Begley TP. Pyridoxal phosphate: Biosynthesis and catabolism. Biochim Biophys Acta - Proteins Proteomics (2011) 1814:1585-1596. doi:10.1016/j.bbapap.2011.06.018

38. Ehrenshaft M, Bilski P, Li M, Chignell CF, Daub ME. A highly conserved sequence is a novel gene involved in de novo vitamin B6 biosynthesis. Proc Natl Acad Sci U S A (1999) 96:93749378. doi:10.1073/pnas.96.16.9374

39. Neuwirth M, Strohmeier M, Windeisen V, Wallner S, Deller S, Rippe K, Sinning I, Macheroux P, Tews I. X-ray crystal structure of Saccharomyces cerevisiae Pdx1 provides 
insights into the oligomeric nature of PLP synthases. FEBS Lett (2009) 583:2179-2186. doi:10.1016/j.febslet.2009.06.009

448

449

450

451

452

453

454

455

456

457

458

459

460

461

462

463

464

465

466

467

468

469

470

471

472

473

474

475

476

477

478

479

480

481

482

483

40. Smith AM, Brown WC, Harms E, Smith JL. Crystal structures capture three states in the catalytic cycle of a pyridoxal phosphate (PLP) Synthase. J Biol Chem (2015) 290:5226-5239. doi:10.1074/jbc.M114.626382

41. Guédez G, Hipp K, Windeisen V, Derrer B, Gengenbacher M, Böttcher B, Sinning I, Kappes $\mathrm{B}$, Tews I. Assembly of the eukaryotic PLP-synthase complex from Plasmodium and activation of the Pdx1 enzyme. Structure (2012) 20:172-184. doi:10.1016/j.str.2011.11.015

42. Strohmeier M, Raschle T, Mazurkiewicz J, Rippe K, Sinning I, Fitzpatrick TB, Tews I. Structure of a bacterial pyridoxal 5'-phosphate synthase complex. Proc Natl Acad Sci (2006) 103:19284-19289. doi:10.1073/pnas.0604950103

43. Neuwirth M, Flicker K, Strohmeier M, Tews I, Macheroux P. Thermodynamic characterization of the protein-protein interaction in the heteromeric Bacillus subtilis pyridoxalphosphate synthase. Biochemistry (2007) 46:5131-5139. doi:10.1021/bi602602x

44. Grubman A, Phillips A, Thibonnier M, Kaparakis-Liaskos M, Johnson C, Thiberge JM, Radcliff FJ, Ecobichon C, Labigne A, de Reuse H, et al. Vitamin B6 is required for full motility and virulence in Helicobacter pylori. MBio (2010) 1:1-9. doi:10.1128/mBio.00112-10

45. Dick T, Manjunatha U, Kappes B, Gengenbacher M. Vitamin B6 biosynthesis is essential for survival and virulence of Mycobacterium tuberculosis. Mol Microbiol (2010) 78:980-988. doi:10.1111/j.1365-2958.2010.07381.x

46. Qaidi S El, Yang J, Zhang JR, Metzger DW, Bai G. The vitamin B6 biosynthesis pathway in streptococcus pneumoniae is controlled by pyridoxal 5'-phosphate and the transcription factor PdxR and has an impact on ear infection. J Bacteriol (2013) 195:2187-2196. doi:10.1128/JB.00041-13

47. Wrenger C, Knöckel J, Walter RD, Müller IB. Vitamin B1 and B6 in the malaria parasite: Requisite or dispensable? Brazilian J Med Biol Res (2008) 41:82-88. doi:10.1590/S0100879X2008005000006

48. Kronenberger T, Lindner J, Meissner KA, Zimbres FM, Coronado MA, Sauer FM, Schettert I, Wrenger C. Vitamin B6-dependent enzymes in the human malaria parasite plasmodium falciparum: A druggable target? Biomed Res Int (2014) 2014: doi:10.1155/2014/108516

49. Kronenberger T, Schettert I, Wrenger C. Targeting the vitamin biosynthesis pathways for the treatment of malaria. Future Med Chem (2013) 5:769-779. doi:10.4155/fmc.13.43

50. Wrenger C, Eschbach ML, Müller IB, Warnecke D, Walter RD. Analysis of the vitamin B6 biosynthesis pathway in the human malaria parasite Plasmodium falciparum. J Biol Chem (2005) 280:5242-5248. doi:10.1074/jbc.M412475200

51. Zhu J, Burgner JW, Harms E, Belitsky BR, Smith JL. A new arrangement of $(\beta / \alpha) 8$ barrels in the synthase subunit of PLP synthase. J Biol Chem (2005) 280:27914-27923.

doi:10.1074/jbc.M503642200 
52. Raschle T, Amrhein N, Fitzpatrick TB. On the two components of pyridoxal 5'-phosphate synthase from Bacillus subtilis. J Biol Chem (2005) 280:32291-32300. doi:10.1074/jbc.M501356200

487 53. Newman JA, Das SK, Sedelnikova SE, Rice DW. Cloning, purification and preliminary crystallographic analysis of a putative pyridoxal kinase from Bacillus subtilis. Acta Crystallogr Sect F Struct Biol Cryst Commun (2006) 62:1006-1009. doi:10.1107/S1744309106035779

54. Gandhi AK, Desai J V., Ghatge MS, di Salvo ML, Di Biase S, Danso-Danquah R, Musayev FN, Contestabile R, Schirch V, Safo MK. Crystal structures of human pyridoxal kinase in complex with the neurotoxins, ginkgotoxin and theophylline: Insights into pyridoxal kinase inhibition. PLoS One (2012) 7:1-11. doi:10.1371/journal.pone.0040954

55. Barra ALC, Dantas L de OC, Morão LG, Gutierrez RF, Polikarpov I, Wrenger C, Nascimento AS. Essential Metabolic Routes as a Way to ESKAPE from Antibiotic Resistance. bioRxiv (2019)817015. doi:10.1101/817015 
bioRxiv preprint doi: https://doi.org/10.1101/817015; this version posted January 13,2020 . The copyright holder for this preprint (which was not certified by peer review) is the author/funder, who has granted bioRxiv a license to display the preprint in perpetuity. It is made available under aCC-BY-NC 4.0 International license.

Running Title

500 Table 1. Panel of observed enzymes for the thiamine pathway in ESKAPE pathogens.

\begin{tabular}{ccccccc}
\hline Enzyme & $\begin{array}{c}\text { Enterococcus } \\
\text { faecium } \\
\text { (ATCC 8459) }\end{array}$ & $\begin{array}{c}\text { Staphylococcus } \\
\text { aureus } \\
(\text { NCTC 8325) }\end{array}$ & $\begin{array}{c}\text { Klebsiella } \\
\text { pneumoniae } \\
\text { (subsp. } \\
\text { Pneumoniae } \\
\text { ATCC 43816 } \\
\text { KPPR1) }\end{array}$ & $\begin{array}{c}\text { Acinetobacter } \\
\text { baumannii } \\
\text { (ATCC 17978) }\end{array}$ & $\begin{array}{c}\text { Pseudomonas } \\
\text { aeruginosa } \\
\text { (NCGM 1900) }\end{array}$ & $\begin{array}{c}\text { Enterobacter } \\
\text { sp. } \\
(638)\end{array}$ \\
\hline ThiD & $\checkmark$ & $\checkmark$ & $\checkmark$ & $\checkmark$ & $\times$ & $\checkmark$ \\
\hline THiM & $\times$ & $\checkmark$ & $\checkmark$ & $\checkmark$ & $\checkmark$ & $\checkmark$ \\
\hline ThiE & $\times$ & $\checkmark$ & $\checkmark$ & $\checkmark$ & $\times$ & $\times$ \\
\hline TPK & $\checkmark$ & $\checkmark$ & $\checkmark$ & $\times$ & $\times$ & $\times$ \\
\hline TenA & $\times$ & $\checkmark$ & $\checkmark$ & $\checkmark$ & $\checkmark$ & $\times$ \\
\hline
\end{tabular}

501 
bioRxiv preprint doi: httos://doi org/101101/817015: this version posted January 13,2020 . The copvriaht holder for this preprint (which was not certified by peer review) is the author/funder, who has granted bioRxiv a license to display the preprint in perpetuity. It is made available under aCC-BY-NC 4.0 Internationtaliphipf Biosynthesis Pathways in Pathogens

502 Table 2. Panel of observed enzymes for the pyridoxal 5'-phosphate pathway in ESKAPE 503 pathogens.

\begin{tabular}{ccccccc}
\hline Enzyme & $\begin{array}{c}\text { Enterococcus } \\
\text { faecium } \\
\text { (ATCC 8459) }\end{array}$ & $\begin{array}{c}\text { Staphylococcus } \\
\text { aureus } \\
(\text { NCTC 8325) }\end{array}$ & $\begin{array}{c}\text { Klebsiella } \\
\text { pneumoniae } \\
(\text { subsp. } \\
\text { Pneumoniae } \\
\text { ATCC 43816 } \\
\text { KPPR1) }\end{array}$ & $\begin{array}{c}\text { Acinetobacter } \\
\text { baumannii } \\
(\text { ATCC 17978) }\end{array}$ & $\begin{array}{c}\text { Pseudomonas } \\
\text { aeruginosa } \\
(\text { NCGM 1900) }\end{array}$ & $\begin{array}{c}\text { Enterobacter } \\
\text { sp. } \\
(638)\end{array}$ \\
\hline Pdx1 & $\mathbf{x}$ & $\checkmark$ & $\mathbf{x}$ & $\mathbf{x}$ & $\mathbf{x}$ & $\mathbf{x}$ \\
\hline Pdx2 & $\mathbf{x}$ & $\checkmark$ & $\mathbf{x}$ & $\mathbf{x}$ & $\mathbf{x}$ & $\mathbf{x}$ \\
\hline
\end{tabular}

504 


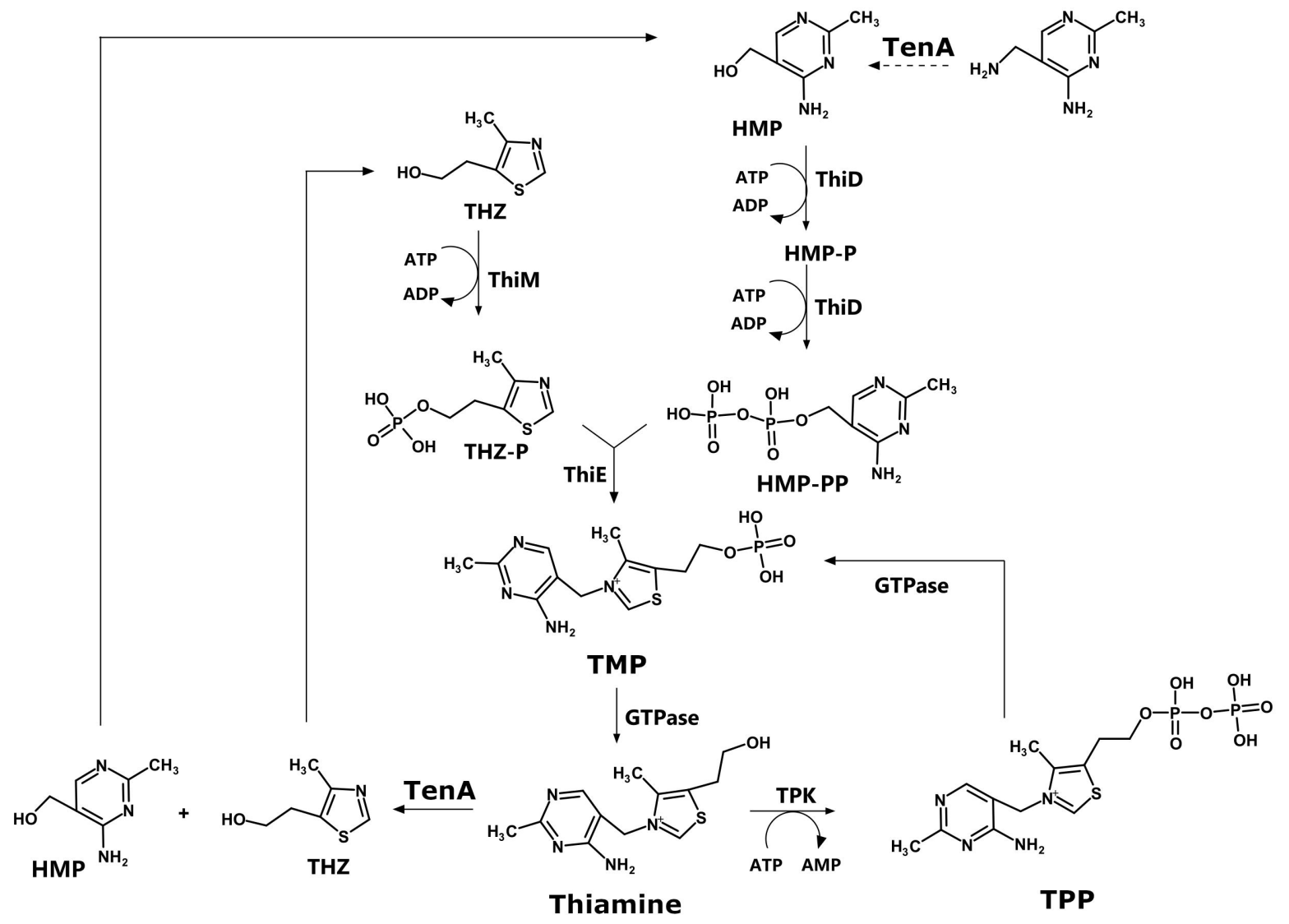




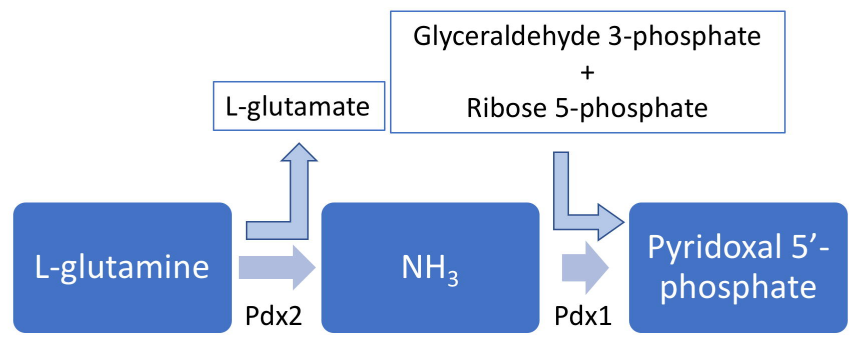

